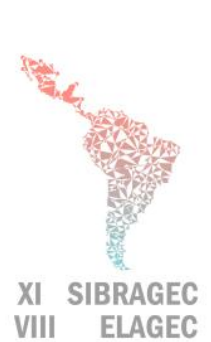

VIII ELAGEC

\section{SIMPÓSIO BRASILEIRO DE GESTÃO E ECONOMIA DA CONSTRUÇÃO \\ VIII ENCUENTRO LATINOAMERICANO DE GESTIÓN \\ Y ECONOMÍA DE LA CONSTRUCCIÓN}

Do conhecimento à ação: práticas avançadas de gestão da produção

Londrina, Paraná, Brasil. 23 a 25 de Outubro de 2019

\title{
GERENCIAMENTO DE RESÍDUOS DA CONSTRUÇÃO COM BIM: UMA EXPLORAÇÃO SOBRE A REALIDADE ATUAL
}

\author{
MIARA, Renata Degraf (1); TAVARES, Sergio F. (2); SCHEER, Sergio (3)
}

(1) UFPR, (41) 99903-8268, e-mail: renata.miara@ufpr.br (2) UFPR, e-mail: sergioftavares@gmail.com, (3) UFPR, e-mail: scheer@ufpr.br

\begin{abstract}
Minimizing construction waste is one of the major challenges of the sector in our country. Estimating the losses and waste generated in civil construction accurately is vital for minimizing them in the project stage. This vision allows engineers to visualize and explore possible corrective measures in order to increase construction efficiency. In addition, it can enable builders to identify critical processes in waste generation and plan control strategies. By integrating several projects into a single model, the process of modeling the construction information (BIM) can assist in the solution of this challenge. Through a questionnaire applied to engineers and architects, this work intends to explore the current reality and the potential of the application of BIM in the generation of construction waste.
\end{abstract}

Keywords: BIM, Waste, Construction, Economy, Efficiency.

\section{INTRODUÇÃO}

A modelagem da informação da construção (BIM) é um dos mais recentes desenvolvimentos na indústria da arquitetura, engenharia e construção (AEC). Através do uso da tecnologia BIM, um modelo digital acurado da edificação é construído (AZHAR, 2011).

Um modelo BIM proporciona a superposição de projetos de diferentes disciplinas em um único modelo criando oportunidades para incorporar a sustentabilidade dentro do processo de projeto. Este modelo pode ser utilizado para a visualização dos impactos das diferentes alternativas de projeto na sustentabilidade do edifício, proporcionando aos projetistas um processo de decisão mais racional.

Um dos principais aspectos relacionados à sustentabilidade de uma edificação é a geração dos resíduos ligados aos processos de construção e demolição. Esses resíduos, geralmente chamados de resíduos da construção e demolição (C\&D) representam grande parte do material destinado aos aterros. Na China, aproximadamente 1,13 bilhões de toneladas de resíduos de C\&D foram gerados em 2014 (LU et al., 2016). No Brasil foram coletados 45,1 milhões de toneladas de resíduos da C\&D no ano de 2016 (ABRELPE, 2016). No Reino Unido 32\% do material destinado aos aterros é resíduo proveniente da C\&D (PRISM ENVIRONMENT, 2012). A indústria da construção é responsável por $25-30 \%$ de todos os resíduos gerados na União Europeia (COMISSION, 2015) e de $20 \%$ no Japão (MOE, 2014). 
Com o objetivo de objetivo de determinar quais as principais barreiras que impedem um gerenciamento adequado dos resíduos da construção este estudo se propõe na realização de um questionário. Os resultados obtidos servirão de forma a explorar a realidade atual do gerenciamento de resíduos da construção e do uso dos processos BIM dentre profissionais da indústria.

\section{MÉTODO}

Este estudo teve como método a realização de uma survey, ou questionário. Este questionário teve como principal objetivo a determinação do nível de conhecimento e prática atual a respeito do gerenciamento de resíduos da construção civil e dos processos BIM. Buscou-se também avaliar como esses profissionais acreditam que uma quantificação na fase de projeto refletirá na redução e mitigação desses resíduos.

O questionário teve como público-alvo engenheiros e arquitetos atuantes nas fases de projeto, planejamento e execução de obras civis. Ele foi realizado de maneira online, a partir de formulário elaborado no Google Forms.

Os dados levantados foram analisados com técnicas próprias para a pesquisa qualitativa, visto que o processo e seu significado são os focos principais dessa abordagem. $\mathrm{O}$ foco foi dado na análise de conteúdo, e por esta razão não foi realizado tratamento estatístico. Esse tipo de análise tem como objetivo inferir conclusões acerca do conteúdo buscando diminuir a subjetividade comum as pesquisas qualitativas (DRESCH et al., 2015).

\section{DESENVOLVIMENTO}

O questionário foi estruturado de forma a abrangir três aspectos: experiência em BIM, percepção de resíduos e minimização dos mesmos.

Sua estrutura foi baseada em O'Reilly (2012). Neste artigo o autor desenvolveu uma análise qualitativa através de questionário para arquitetos e engenheiros do Reino Unido com o mesmo objetivo proposto. A estrutura utilizada para explorar a realidade brasileira está apresentada no Apêndice.

O questionário foi enviado por e-mail para 55 empresas da região de Curitiba, capital do Estado do Paraná. As empresas entrevistadas incluíram empresas de engenharia e arquitetura que realizam projetos e/ou acompanham a execução de obras.

Além disso, o questionário foi enviado para grupos de e-mails dos cursos de Engenharia Civil e Arquitetura da UFPR com o objetivo de atingir ex-alunos garantindo uma maior gama de profissionais da área.

Este universo de pesquisa foi definido com o objetivo de abrangir o maior número de profissionais possíveis na cidade de Curitiba. As empresas foram buscadas através do site Google, utilizando as palavras-chave: "empresa de engenharia" e "Curitiba"; "construtora" e "Curitiba"; "escritório de arquitetura" e "Curitiba"; "escritório de projetos" e "Curitiba"; "projetos em BIM" e "Curitiba".

A análise do questionário pode ser dividida em algumas etapas, relatadas a seguir:

\subsection{Identificação dos respondentes}

O número de respostas obtidas foi 46. Deste total, 29 foram Engenheiros Civis, 5 Arquitetos e 1 Consultor em Tecnologia BIM. Os 11 restantes foram outros 
SIBRAGEC - ELAGEC 2019 - de 23 a 25 de Outubro - LONDRINA - PR

profissionais relacionados à projetos, execução e acompanhamento de empreendimentos de engenharia e arquitetura.

$39,1 \%$ dos respondentes trabalham em escritórios de projetos, enquanto $37 \%$ trabalham em construtoras. A grande maioria das empresas nas quais os profissionais trabalham podem ser consideradas como pequenas empresas, visto que o número de funcionários indicado em 54,8\% das respostas foi entre 1 e 10.

\subsection{Utilização do BIM}

Na pergunta que correspondia à utilização de BIM nos projetos em que atua, metade dos entrevistados respondeu que utiliza. Em relação à auto-avaliação do conhecimento a respeito de BIM, a maioria dos respondentes avaliou seu conhecimento como básico (37\%), seguido por intermediário $(34,8 \%)$, avançado $(19,6 \%)$ e desconhecimento do BIM $(8,7 \%$ ou 4 respostas).

Para correta análise das próximas respostas do questionário foram então desconsiderados aqueles respondentes que alegaram desconhecer o BIM, resultando então em 42 respostas válidas.

Grande parte dos respondentes (41 entre 42 - 97,6\%) acredita que o BIM possa ser utilizado como um processo para redução dos resíduos gerados na construção. 40 respondentes $(95,2 \%)$ acreditam que o arquiteto e/ou engenheiro projetista têm papel significante na possibilidade de redução dos resíduos gerados.

\subsection{Quantificação dos resíduos da construção}

31 respondentes $(73,8 \%)$ acreditam que quantificar os resíduos gerados na construção, durante o projeto, pode ajudar a reduzi-los de maneira geral., enquanto os 11 restantes $(26,2 \%)$ acreditam que essa redução talvez poderia acontecer.

A pergunta seguinte foi então sobre em qual medida ocorreria essa redução. Metade dos respondentes acredita que esta redução se dará em média proporção. Em relação à consideração da geração de resíduos no último projeto realizado, $33(78,6 \%)$ responderam que esta não foi considerada. No entanto foi surpreendente verificar que 9 dos 42 respondentes $(21,4 \%)$ já consideraram esse aspecto em seus últimos projetos, visto que era de se esperar que ninguém o houvesse feito.

\subsection{Noções a respeito da geração de resíduos}

Com o objetivo de avaliar como está o conhecimento em relação à geração de resíduos dos profissionais de engenharia e arquitetura, foi pedido para que os profissionais classificassem os sistemas de estruturas e de vedação conforme a geração de resíduos. A escala utilizada para tal avaliação consistiu em números de 1 a 5, em que 5 significava um sistema com alta geração de resíduos e 1 um sistema com baixa geração dos mesmos.

Os sistemas avaliados foram:

* Estrutural: metálica, concreto armado, concreto pré-moldado;

* Vedação: dry-wall, alvenaria convencional, wood-frame e alvenaria estrutural.

O Quadro 1 indica o número de respostas referente à cada número da escala para os sistemas estruturais e o Gráfico 1 ilustra de forma gráfica estes resultados. 
SIBRAGEC - ELAGEC 2019 - de 23 a 25 de Outubro - LONDRINA - PR

Quadro 1 - Avaliação quanto à geração de resíduos dos sistemas

\begin{tabular}{|c|c|c|c|c|c|}
\hline Sistemas estruturais & $\mathbf{1}$ & $\mathbf{2}$ & $\mathbf{3}$ & $\mathbf{4}$ & $\mathbf{5}$ \\
\hline Metálica & 19 & 13 & 9 & 0 & 1 \\
\hline Concreto-armado & 2 & 2 & 6 & 7 & 25 \\
\hline Concreto pré-moldado & 8 & 14 & 14 & 6 & 0 \\
\hline
\end{tabular}

\section{Gráfico 1 - Avaliação quanto à geração de resíduos}

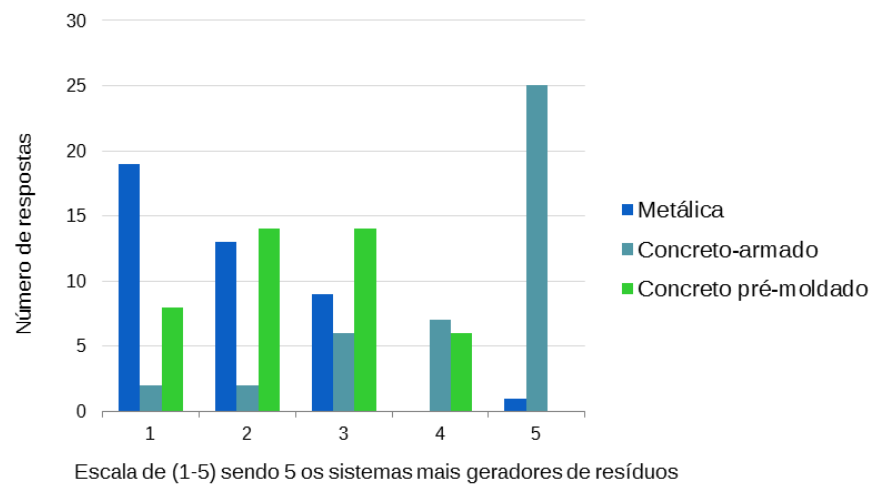

Para os sistemas de vedação os resultados são ilustrados no Quadro 2 e no Erro! Fonte de referência não encontrada.2. A partir das tabelas e gráficos verifica-se que o nível de conhecimento em relação à geração de resíduos dos sistemas está de acordo.

Os sistemas estruturais foram classificados conforme a geração de resíduos na seguinte ordem: concreto armado, concreto pré-moldado e estrutura metálica (do sistema que gera mais resíduos para aquele que gera menos).

Enquanto que os sistemas de vedação foram classificados na seguinte ordem: alvenaria convencional, alvenaria estrutural, wood-frame e dry-wall.

\section{Quadro 2 - Avaliação quanto à geração de resíduos dos sistemas}

\begin{tabular}{|c|c|c|c|c|c|}
\hline Sistemas de vedação & $\mathbf{1}$ & $\mathbf{2}$ & $\mathbf{3}$ & $\mathbf{4}$ & $\mathbf{5}$ \\
\hline Dry-wall & 5 & 13 & 16 & 4 & 4 \\
\hline Alvenaria convencional & 0 & 2 & 5 & 12 & 23 \\
\hline Wood-frame & 6 & 12 & 15 & 9 & 0 \\
\hline Alvenaria estrutural & 0 & 8 & 11 & 18 & 5 \\
\hline
\end{tabular}

Gráfico 1 - Avaliação quanto à geração de resíduos

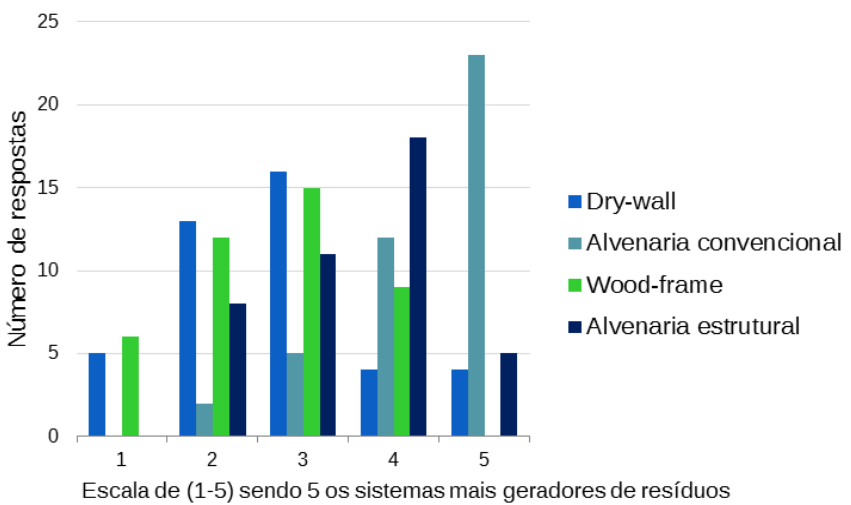


Em relação à em quais etapas do projeto a geração de resíduos deveria ser considerada, as respostas se mostraram equilibradas, no sentido de que este aspecto deveria ser considerado em todas as fases: estudo preliminar, ante-projeto, projeto legal e projeto executivo.

\subsection{Barreiras para implantação}

Com o objetivo de determinar quais as principais barreiras que impedem que um gerenciamento adequado dos resíduos da construção, foi pedido que os respondentes classificassem novamente em uma escala de 1-5 as barreiras, sendo com nota 5 aquelas mais impactantes.

O Quadro 3 e o Gráfico 3 ilustram os resultados obtidos. Segundo os respondentes a barreira mais impactante é o próprio aspecto cultural do setor da construção civil, seguido pela falta de incentivos financeiros e na dificuldade da obtenção de dados de geração de resíduos.

Quadro 3 - Avaliação das barreiras no gerenciamento de resíduos

\begin{tabular}{|c|c|c|c|c|c|}
\hline Barreiras & $\mathbf{1}$ & $\mathbf{2}$ & $\mathbf{3}$ & $\mathbf{4}$ & $\mathbf{5}$ \\
\hline Preocupação com outros aspectos do projeto & 3 & 7 & 13 & 9 & 10 \\
\hline Falta de incentivos financeiros & 4 & 7 & 8 & 11 & 12 \\
\hline Falta de legislação & 7 & 9 & 10 & 10 & 6 \\
\hline Restrição de tempo & 4 & 8 & 11 & 14 & 5 \\
\hline $\begin{array}{c}\text { Dados de geração de resíduos são muito difíceis } \\
\text { de se obter }\end{array}$ & 2 & 8 & 10 & 11 & 11 \\
\hline Falta de motivação pessoal & 4 & 7 & 14 & 13 & 4 \\
\hline Cultura do setor & 1 & 1 & 7 & 12 & 21 \\
\hline
\end{tabular}

Gráfico 3 - Avaliação das barreiras no gerenciamento de resíduos

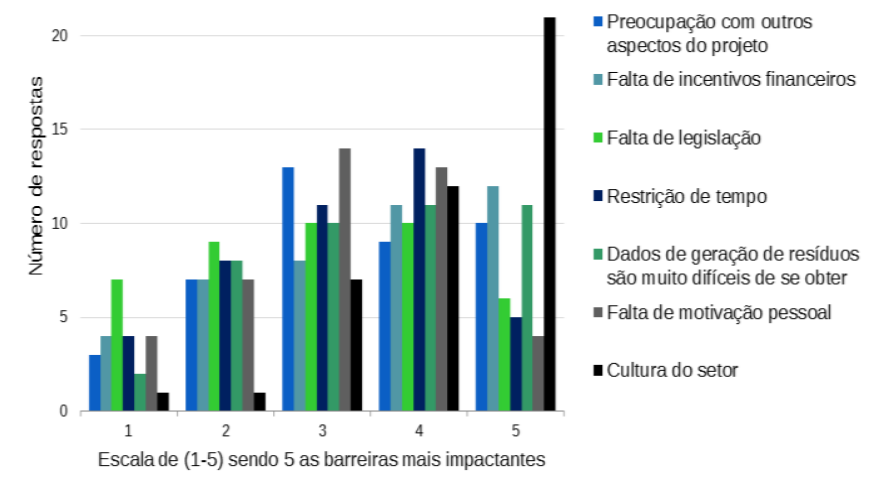

\subsection{Aplicação de Plug-in BIM}

A última pergunta feita no questionário foi em relação à aplicação de um plug-in em software BIM para gerenciamento dos resíduos da construção. $85 \%$ dos respondentes avaliaram que um plug-in que extraísse do modelo os quantitativos de geração de 
SIBRAGEC - ELAGEC 2019 - de 23 a 25 de Outubro - LONDRINA - PR

resíduos, ao longo do cronograma da obra, poderia sim reduzir a geração dos mesmos de maneira geral.

\section{CONCLUSÃO}

Assim como o estudo realizado por O'Reilly (2012), este estudo evidenciou a potencial aplicação do BIM como ferramenta para redução de geração de resíduos. A principal característica que determinou este resultado foi à possibilidade de se obter informações a respeito dos resíduos durante a etapa de projeto, proporcionando aos arquitetos e engenheiros a tomada de decisões com base em informações.

A partir do estudo pode-se também verificar que as estratégias de gerenciamento e minimização dos resíduos devem ser consideradas de maneira holística, ao longo de todo o ciclo de vida do projeto.

Verificou-se também que os arquitetos e engenheiros apresentam boa percepção em relação à geração de resíduos e entendem sua responsabilidade sob este aspecto, no entanto, são outras as barreiras que restringem a capacidade de redução.

O objetivo principal proposto foi atingido, ao serem identificadas as principais barreiras no gerenciamento de resíduos. As principais barreiras identificadas, como: falta de incentivos financeiros, dados de geração muito difíceis de obter e barreira cultural do setor, poderiam ser vencidas através do desenvolvimento de uma ferramenta capaz de quantificar a geração de resíduos na etapa de projeto.

Um aplicativo, integrado ao modelo BIM, capaz de extrair por material o volume de resíduos gerados dando como resultado uma estimativa detalhada da geração de resíduos no projeto poderia reduzir significativamente o volume de resíduos gerados.

\section{REFERÊNCIAS}

ABRELPE. Panorama dos resíduos sólidos no Brasil 2016. Brasília: Associação Brasileira de Limpeza Pública e Resíduos Especiais, 2016. Disponível em:

<http://www.abrelpe.org.br/Panorama/panorama2016.pdf>. Acesso em: 03 maio 2018.

AJAYI, Saheed O. et al. Waste effectiveness of the construction industry: Understanding the impediments and requisites for improvements. Resources, Conservation And Recycling, [s.1.], v. 102, p.101-112, set. 2015. Elsevier BV. http://dx.doi.org/10.1016/j.resconrec.2015.06.001.

AKANBI, Lukman A. et al. Salvaging building materials in a circular economy: A BIM-based whole-life performance estimator. Resources, Conservation And Recycling, [s.1.], v. 129, p.175-186, fev. 2018. Elsevier BV. http://dx.doi.org/10.1016/j.resconrec.2017.10.026.

AKINADE, Olugbenga O. et al. Designing out construction waste using BIM technology: Stakeholders' expectations for industry deployment. Journal Of Cleaner Production, [s.l.], v. 180, p.375-385, abr. 2018. Elsevier BV. http://dx.doi.org/10.1016/j.jclepro.2018.01.022.

AKINADE, Olugbenga O. et al. Evaluation criteria for construction waste management tools: towards a holistic BIM framework. International Journal Of Sustainable Building Technology And Urban Development, [s.1.], v. 7, n. 1, p.3-21, 2 jan. 2016. Informa UK Limited. http://dx.doi.org/10.1080/2093761x.2016.1152203.

AKINADE, Olugbenga O. et al. Waste minimisation through deconstruction: A BIM based Deconstructability Assessment Score (BIM-DAS). Resources, Conservation And Recycling, [s.1.], v. 105, p.167-176, dez. 2015. Elsevier BV.

http://dx.doi.org/10.1016/j.resconrec.2015.10.018. 


\section{SIBRAGEC - ELAGEC 2019 - de 23 a 25 de Outubro - LONDRINA - PR}

BARBETTA, Pedro A. Estatística Aplicada às Ciências Sociais. 5.ed. Florianópolis: Ed. UFSC, 2006.

BILAL, Muhammad et al. Analysis of critical features and evaluation of BIM software: towards a plug-in for construction waste minimization using big data. International Journal Of Sustainable Building Technology And Urban Development, [s.1.], v. 6, n. 4, p.211-228, 2 out. 2015. Informa UK Limited. http://dx.doi.org/10.1080/2093761x.2015.1116415.

BILAL, Muhammad et al. Big data architecture for construction waste analytics (CWA): A conceptual framework. Journal Of Building Engineering, [s.1.], v. 6, p.144-156, jun. 2016. Elsevier BV. http://dx.doi.org/10.1016/j.jobe.2016.03.002.

BIM COMITTEE. NBIMS: National Building Information Modeling Standard. Part 1 ed. Washigton: Us National Institute Of Building Sciences Facilities Information Council, 2007.

CHENG, Jack C.p.; MA, Lauren Y.h.. A BIM-based system for demolition and renovation waste estimation and planning. Waste Management, [s.1.], v. 33, n. 6, p.1539-1551, jun. 2013. Elsevier BV. http://dx.doi.org/10.1016/j.wasman.2013.01.001.

CHENG, Jack C.p.; WON, Jongsung; DAS, Moumita. Construction and demolition waste management using BIM technology. In: CONF. OF THE INT'L. GROUP FOR LEAN CONSTRUCTION, 23., 2015, Perth. Proceedings.... Australia: Glc, 2015. p. 1 - 12.

COMISSION, European. Construction and Demolition Waste (CDW). Bruxelas: Ec, 2015. Disponível em: <http://ec.europa.eu/environment/waste/construction_demolition.htm>. Acesso em: 30 abr. 2018.

DRESCH, Aline; LACERDA, Daniel Pacheco; ANTUNES JÚNIOR, José Antonio Val-le. Design Science Research. São Paulo: Bookman, 2015.

GE, Xin Janet et al. Deconstruction waste management through 3d reconstruction and bim: a case study. Visualization In Engineering, [s.1.], v. 5, n. 1, p.1-15, 14 jul. 2017. Springer Nature. http://dx.doi.org/10.1186/s40327-017-0050-5.

GHAFAR, M. Abdul; IBRAHIM, R.; SHARI, Z.. Embedding Cultural Knowledge in Building Information Modeling (BIM) for Fabrication Efficiency to Reduce Industrialized Con-struction Waste. Computing In Civil And Building Engineering (2014), [s.l.], p.1-8, 17 jun. 2014. American Society of Civil Engineers. http://dx.doi.org/10.1061/9780784413616.025.

HAMIDI, Behzad et al. Potential Application of BIM in Cost-Benefit Analysis of Demolition Waste Management. Construction Research Congress 2014, [s.1.], p.1-10, 13 maio 2014. American Society of Civil Engineers. http://dx.doi.org/10.1061/9780784413517.029.

KIM, Young-chan et al. An estimation framework for building information modeling (BIM)based demolition waste by type. Waste Management \& Research, [s.1.], v. 35, n. 12, p.12851295, 27 out. 2017. SAGE Publications. http://dx.doi.org/10.1177/0734242x17736381.

LIU, Hexu et al. BIM-based automated design and planning for boarding of light-frame residential buildings. Automation In Construction, [s.1.], v. 89, p.235-249, maio 2018. Elsevier BV. http://dx.doi.org/10.1016/j.autcon.2018.02.001.

LIU, Zhen et al. A BIM-aided construction waste minimisation framework. Automation In Construction, [s.1.], v. 59, p.1-23, nov. 2015. Elsevier BV. http://dx.doi.org/10.1016/j.autcon.2015.07.020.

LU, Weisheng et al. Estimating and calibrating the amount of building-related construction and demolition waste in urban China. International Journal Of Construction Management, [s.1.], v. 17, n. 1, p.13-24, 25 abr. 2016. Informa UK Limited. http://dx.doi.org/10.1080/15623599.2016.1166548. 
MOE (Org.). History and Current State of Waste Management in Japan. Tóquio: Moe, 2014. Disponível em: 〈http://www.env.go.jp/en/recycle/smcs/attach/hcswm.pdf〉. Acesso em: 30 abr. 2018.

O'REILLY, Alistair. Using BIM as a tool for cutting construction waste at source.

Construction Research And Innovation, [s.1.], v. 3, n. 1, p.28-31, mar. 2012. Informa UK Limited. http://dx.doi.org/10.1080/20450249.2012.11873828.

PORWAL, Atul; HEWAGE, Kasun N.. Building Information Modeling-Based Analysis to Minimize Waste Rate of Structural Reinforcement. Journal Of Construction Engineering And Management, [s.1.], v. 138, n. 8, p.943-954, ago. 2012. American Society of Civil Engineers (ASCE). http://dx.doi.org/10.1061/(asce)co.1943-7862.0000508.

PRISM ENVIRONMENT (United Kingdom). Construction sector overview in the UK. London: Prism Environment, 2012.

WEI, Haitao et al. BIM-based method calculation of auxiliary materials required in housing construction. Automation In Construction, [s.1.], v. 78, p.62-82, jun. 2017. Elsevier BV. http://dx.doi.org/10.1016/j.autcon.2017.01.022.

WON, Jongsung; CHENG, Jack C.p.. Identifying potential opportunities of building information modeling for construction and demolition waste management and minimization. Automation In Construction, [s.1.], v. 79, p.3-18, jul. 2017. Elsevier BV. http://dx.doi.org/10.1016/j.autcon.2017.02.002.

WON, Jongsung; CHENG, Jack C.p.; LEE, Ghang. Quantification of construction waste prevented by BIM-based design validation: Case studies in South Korea. Waste Management, [s.l.], v. 49, p.170-180, mar. 2016. Elsevier BV.

http://dx.doi.org/10.1016/j.wasman.2015.12.026.

WONG, Johnny Kwok Wai; ZHOU, Jason. Enhancing environmental sustainability over building life cycles through green BIM: A review. Automation In Construction, [s.1.], v. 57, p.156-165, set. 2015. Elsevier BV. http://dx.doi.org/10.1016/j.autcon.2015.06.003.

\section{AGRADECIMENTOS}

Agradecemos ao Programa de Pós-Graduação em Engenharia da Construção Civil da Universidade Federal do Paraná (PPGECC-UFPR) e à Universidade Federal do Paraná por proporcionarem a realização deste trabalho e por fomentarem a pesquisa científica no Brasil. Agradecemos também aos professores, deste programa e desta Universidade, por orientarem e colaborarem na realização desse trabalho. 
SIBRAGEC - ELAGEC 2019 - de 23 a 25 de Outubro - LONDRINA - PR

APÊNDICE

\begin{tabular}{l}
\hline Questionário: $\quad$ BIM como ferramenta para redução dos resíduos da construção civil \\
Esta pesquisa faz parte de uma dissertação de mestrado do Programa de Pós-Graduação em \\
Engenharia da Construção Civil, sub-área de Gestão, da Universidade Federal do Paraná \\
(PPGECC) e tem como objetivo explorar a realidade atual do gerenciamento de resíduos da \\
construção e do uso dos processos BIM. O questionário tem como público-alvo engenheiros e \\
arquitetos atuantes nas fases de projeto, planejamento e execução de obras civis.
\end{tabular}

Endereço de e-mail:

Formação:
( )
Engenheiro(a) Civil
( )
Arquiteto(a)
( )
Técnico(a) em edificações
( )
Outros:

Atuação profissional:
( )
Construtora
( )
Escritório de projetos
( )
Empresa de demolição
( )
Empresa de coleta e destinação dos resíduos da construção civil
( )
Profissional autônomo

Nome da empresa em que atua:

Número de funcionários da empresa em que atua:

$\begin{array}{ll}\text { ( ) } & 1-10 \\ \text { ( ) } & 11-25 \\ \text { ( ) } & 26-50 \\ \text { ( ) } & 51-100 \\ \text { ( ) } & \text { Mais de } 100\end{array}$

Anos de experiência do profissional no mercado
( )
$0-5$
( )
6-10
( ) 11-15
( ) Mais de 15

Cidade em que o profissional atua: 
SIBRAGEC - ELAGEC 2019 - de 23 a 25 de Outubro - LONDRINA - PR

Você utiliza o BIM em seus projetos?

$\begin{array}{ll}\text { ( ) } & \text { Sim } \\ \text { ( ) } & \text { Não }\end{array}$

Como você avalia seu conhecimento a respeito de BIM?

$\begin{array}{ll}\text { ( ) } & \text { Nível básico } \\ \text { ( ) } & \text { Nível intermediário } \\ \text { ( ) } & \text { Nível avançado } \\ & \text { Desconheço o BIM }\end{array}$

Você acredita que o BIM pode ser adotado como um processo para redução dos resíduos gerados durante a construção?
( )
Sim
( )
Não

Você acredita que o arquiteto e/ou engenheiro projetista tem um papel significante na redução dos resíduos gerados?

$\begin{array}{ll}\text { ( ) } & \text { Sim } \\ \text { ( ) } & \text { Não } \\ \text { ( ) } & \text { Talvez }\end{array}$

Você acredita que quantificar resíduos da construção na etapa de projeto pode ajudar a reduzir os resíduos de uma obra de maneira geral?
( ) Sim
( ) Não
( ) Talvez

Em que medida você acha que ocorreria essa redução?

$\begin{array}{ll}\text { ( ) } & \text { Muito baixo } \\ \text { ( ) } & \text { Baixo } \\ \text { ( ) } & \text { Médio } \\ \text { ( ) } & \text { Alto } \\ & \text { Muito alto }\end{array}$

A geração de resíduos foi considerada em seu último projeto?
( )
Sim
( )
Não 
SIBRAGEC - ELAGEC 2019 - de 23 a 25 de Outubro - LONDRINA - PR

Classifique os seguintes sistemas conforme a geração de resíduos na etapa de construção:

Escala de (1-5) sendo 5 os sistemas mais geradores de resíduos

\begin{tabular}{|c|c|c|c|c|c|}
\hline Sistemas estruturais & 1 & 2 & 3 & 4 & 5 \\
\hline \multicolumn{6}{|l|}{ Metálica } \\
\hline \multicolumn{6}{|l|}{ Concreto-armado } \\
\hline \multicolumn{6}{|l|}{ Concreto pré-moldado } \\
\hline Sistemas de vedação & 1 & 2 & 3 & 4 & 5 \\
\hline \multicolumn{6}{|l|}{ Dry-wall } \\
\hline \multicolumn{6}{|l|}{ Alvenaria convencional } \\
\hline \multicolumn{6}{|l|}{ Wood-frame } \\
\hline Alvenaria estrutural & & & & & \\
\hline
\end{tabular}

Em quais etapas do projeto você acredita que a geração de resíduos deve ser considerada?
( )
Estudo preliminar
( )
Ante projeto
( )
Projeto legal
( )
Projeto executivo

Classifique as principais barreiras no gerenciamento dos resíduos da construção:

Escala de (1-5) sendo 5 para as barreiras mais impactantes

\begin{tabular}{|c|l|l|l|l|l|}
\hline Barreiras & 1 & 2 & 3 & 4 & 5 \\
\hline Preocupação com outros aspectos do projeto & & & & & \\
\hline Falta de incentivos financeiros & & & & & \\
\hline Falta de legislação & & & & & \\
\hline Restrição de tempo & & & & & \\
\hline $\begin{array}{c}\text { Dados de geração de resíduos são muito difíceis } \\
\text { de se obter }\end{array}$ & & & & & \\
\hline Falta de motivação pessoal & & & & & \\
\hline
\end{tabular}

Você acredita que um plug-in em software BIM com quantitativos de resíduos aliado ao cronograma de obra poderia auxiliar na redução dos resíduos gerados?
( )
Sim
( )
Não
( )
Talvez 